\title{
Periodontal disease and oral hygiene benefits in HIV seropositive and AIDS patients
}

\author{
Silvinha S. S. Lemos ${ }^{1}$, Flávia A. Oliveira ${ }^{2}$, Eneida F. Vencio ${ }^{3}$ \\ ${ }^{1}$ DDS, Department of Oral Pathology, School of Dentistry, Universidade Federal de Goiás (UFG), Goiânia, Goiás, Brazil \\ ${ }^{2}$ Associate Professor, Sector of the General Pathology, Tropical Pathology and Public Health Institute, UFG, Goiânia, Goiás, \\ Brazil \\ ${ }^{3}$ Associate Professor, DDS., Department of Oral Pathology, School of Dentistry, UFG, Brazil
}

\author{
Correspondence: \\ Universidade Federal de Goiás \\ Faculdade de Odontologia \\ Departamento de Patologia Oral \\ Praça Universitária, $s / n$ \\ 74605-220 Goiania, GO Brazil \\ vencio56@hotmail.com
}

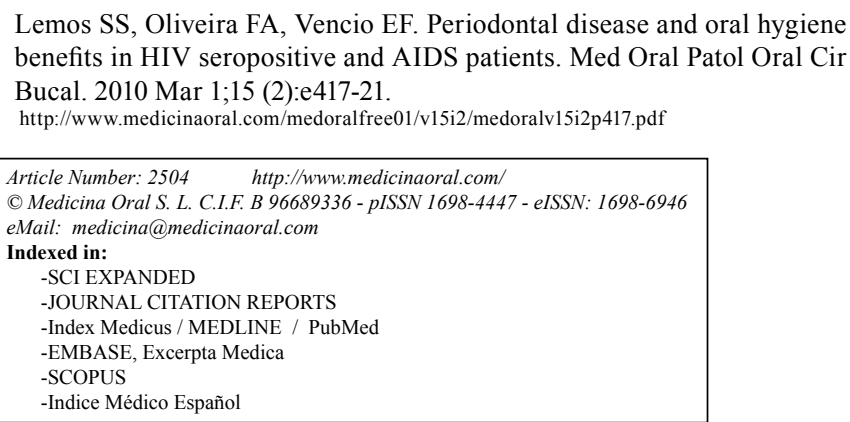

\begin{abstract}
Objectives: The frequency of gingival and periodontal disease in HIV-seropositive and AIDS patients was investigated in order to evaluate the oral hygiene benefits of using mechanical therapy. Study design: thirty-two consenting HIV-positive patients were examined. Their gingival and periodontal status were evaluated using the Gingival Index and the Simplified Oral Hygiene Index. The data were assessed at baseline, after three months and after six months. Subjects received mechanical therapy, which included calculus removal, scaling and root planning, tooth polishing and oral hygiene instructions. The maintenance of oral hygiene was performed weekly. HIV staging and CD4 counts were also investigated. Results: At the baseline, gingival and periodontal disease was present in $71.9 \%$ of all subjects. Chronic gingivitis (43.8\%) was the most frequent in all subjects. A clear improvement in gingival health was registered in $78.2 \%$ of subjects after six months of mechanical therapy. No association was registered between CD4 count and gingival/periodontal status or attachment loss with HIV staging. Conclusions: Chronic gingivitis was the most frequent disease in HIV infected and AIDS patients. Oral hygiene using mechanical therapy improves the gingival condition, suggesting that it is an important step in the maintenance of periodontal health.
\end{abstract}

Key words: AIDS, gingival disease, HIV, mechanical treatment, oral hygiene. 


\section{Introduction}

Chronic gingivitis and periodontitis are the most common forms of gingival and periodontal disease in HIVinfected individuals. The relationship between periodontal health and the prevalence and severity of chronic gingivitis and periodontitis among immunosuppressed HIV infected individuals remains controversial (1). Some authors have demonstrated no significant difference in gingival and periodontal status among the HIV seropositive, those with AIDS, and the general population $(2,3,4)$. Many studies have been conducted in order to detect the frequency of HIV-associated periodontal disease and its prevalence varies from 0 to $47 \%(1,3,5)$. Studies have associated a severe attachment loss or increased periodontal attachment loss in individuals with more advanced stages of AIDS (6). However, an association between periodontal disease and immune status in the HIV-infected individual is not yet clear (7).

Bacterial plaque is generally considered to be the main etiological agent in gingival and periodontal disease (8). Mechanical plaque removal and the maintenance of good oral hygiene are important steps in healing gingivitis (9). In HIV infected patients, periodontal disease usually does not respond to conventional treatment with scaling (1). Adjunctive therapies with chemical agents have been indicated for HIV associated periodontal diseases (10). Studies have shown that the attachment level in HIV seropositive subjects is maintained after scaling and root planning $(2,11,12)$. The purpose of this study was to investigate the periodontal status in HIVseropositive and AIDS patients and to evaluate the oral hygiene benefits using mechanical therapy.

\section{Material and Methods}

In 1996, all the patients who were residents in the Solidarity Center of a Volunteer Organization in Goiânia, Goiás, Brazil, were invited to participate in this study. The inclusion criteria consisted in having at least 20 remaining teeth, receiving antiretroviral therapy, being committed in follow all procedures of the proposal mechanical therapy. Patients with limited compliance were not included in this study. After previous authorization thirty-two patients, 21 male and 11 female, were examined. All subjects were diagnosed as HIV antibody positive by ELISA and confirmed by Western Blot. The subjects were clinically grouped according to the Center for Disease Control (CDC) (13) classification for HIV infection after their medical history was reviewed. This study was conducted following the ethical procedures of the Solidarity Center. As this study was conducted in 1996, the antiretroviral regimen comprised one nucleoside reverse transcriptase inhibitor therapy of one protease inhibitor (14).

The diagnosis of HIV-associated periodontal diseases was conducted based on the EEC Clearinghouse on
Oral Problems Related to HIV Infection (15) in categories, as follow: linear gingival erithema, a distinct red band along the margin of the gingival with integrity of the gingival margin and disproportionately for amount of plaque; necrotizing periodontitis characterized by soft tissue loss as a result of ulceration or necrosis or necrosis with or not bone exposure and pain. Chronic gingivitis was characterized by presence of edema, redness, bleeding on gentle probing or spontaneous loss of integrity of the gingival margin with or no pain with changes in gingival position and texture, and there is an increased tendency to bleeding. When these alterations accomplished with $5 \mathrm{~mm}$ deep of the gingival sulcus, it was defined as chronic periodontitis; health gingivae were clinically intact, with pink color, regular shape, size and contour, intact interdental papillae, and absence of bleeding either spontaneous or gently probed with a periodontal probe. One examiner performed all clinical evaluation, and dental treatment was conducted if necessary.

The periodontal status was recorded using the Gingival Index (GI) and the Simplified Oral Hygiene Index (SOHI). The Gingival Index (16) was used to diagnose gingival inflammation and the Simplified Oral Hygiene Index (17) to quantify supragingival plaque accumulation and calculus. The measurements of gingival sulcus and periodontal pockets were determined according to the Ramjford technique (18).

The oral hygiene protocol consisted of mechanical therapy, oral hygiene instruction and maintenance. After the initial diagnosis and assessment of periodontal parameters, all the patients were submitted to standard mechanical therapy and received instructions regarding oral hygiene (9). The mechanical therapy consisted of the removal of supragingival and subgingival plaque and calculus by scaling and root planning using hand instruments, followed by tooth surface polishing with a rubber cup and pumice. Unpolished and rough amalgam fillings were polished when necessary. All subjects received oral hygiene instructions, including the Bass technique and an appropriate interdental cleaning device with dental floss, including fluoride application at the end of each visit. Oral hygiene instruction was performed weekly and individually to achieve optimal plaque control by self-care. A blue-staining substance was used to control weekly plaque accumulation. Mechanical tooth cleaning was performed when indicated. One examiner performed all the clinical evaluations and dental treatment was conducted when necessary. The registration of probing depths, bleeding upon probing and plaque accumulation was taken at baseline, after three months and finally after six months.

The subjects were motivated in the maintenance of oral hygiene, and received weekly support emphasizing the daily removal of plaque accumulation and the use of 
proper techniques. Furthermore, educational seminars were conducted monthly in the Solidarity Center for approximately 50 minutes on themes such as oral hygiene techniques, periodontal disease, HIV infection, stress and diet. Oral hygiene kits were distributed to all the subjects, which included a soft toothbrush, toothpaste, flat dental floss and a brochure with instructions on oral hygiene technique.

The association between gingival health and oral hygiene, gingival health and HIV infection was established by the Chi-square test or Fisher's exact test. The results were considered statistically significant when $\mathrm{p}<0.05$.

\section{Results}

The thirty-two consenting HIV seropositive patients ranging from 30 to 56 years of age (mean age 32 years old), of whom $66 \%$ were male. According to the CDC classification (13), $71.9 \%$ of the patients were grouped in Groups III and IV (Table 1). At the baseline, gingival and periodontal disease was registered in $71.9 \%$ of all subjects, of which $25 \%$ represented HIV associated disease (Table 1). Chronic gingivitis was the most frequent (43.8\%) with $12.5 \%$ affecting Group III and 25\% Group IV. Periodontitis was registered in just one patient. HIVassociated gingival and periodontal disease was seen in $25 \%$ of patients at baseline, of which $21.8 \%$ belonged to Groups III and IV (Table 1). Necrotizing periodontitis was registered in two patients.

A statistically significant difference in the frequency of gingival health was seen after 3 months of oral hygiene protocol (28.1\% vs. 68.7\%), reaching $78.1 \%$ at the end of the study (Table 2). No improvement was registered for linear gingival erythema $(p>0.05)$. Improvement in the clinical aspects of periodontitis and necrotizing periodontitis and statistically significant improvement of the chronic gingivitis was registered at the end of this study (Table 2). The improvement in gingival health was registered after 6 months in patients from Groups I and II in comparison to Groups III and IV. After six months, $78.2 \%$ of the patients presented healthy gingival, with $53.2 \%$ from Groups III and IV (Table 1).

The frequency of the Gingival Index $(0.0-0.1)$ and the Simplified Oral Hygiene Index $(0.0-1.2)$ were significantly higher at the end of this study, with $78.1 \%$ vs. $28.1 \%$ and $87.6 \%$ vs. $34.4 \%$, respectively, $\mathrm{p}<0.05$ (Table 2). No significant difference was registered between the CD4 count and periodontal gingival health $\mathrm{p}>0.05$. Gingival health was registered in subjects with low CD4 counts $(<500$ cells $/ \mathrm{mm} 3)$ after six months of mechanical therapy. No statistically significant association was found between bleeding on probing, pocket formation or attachment loss with CD4 counts among HIV infected individuals.

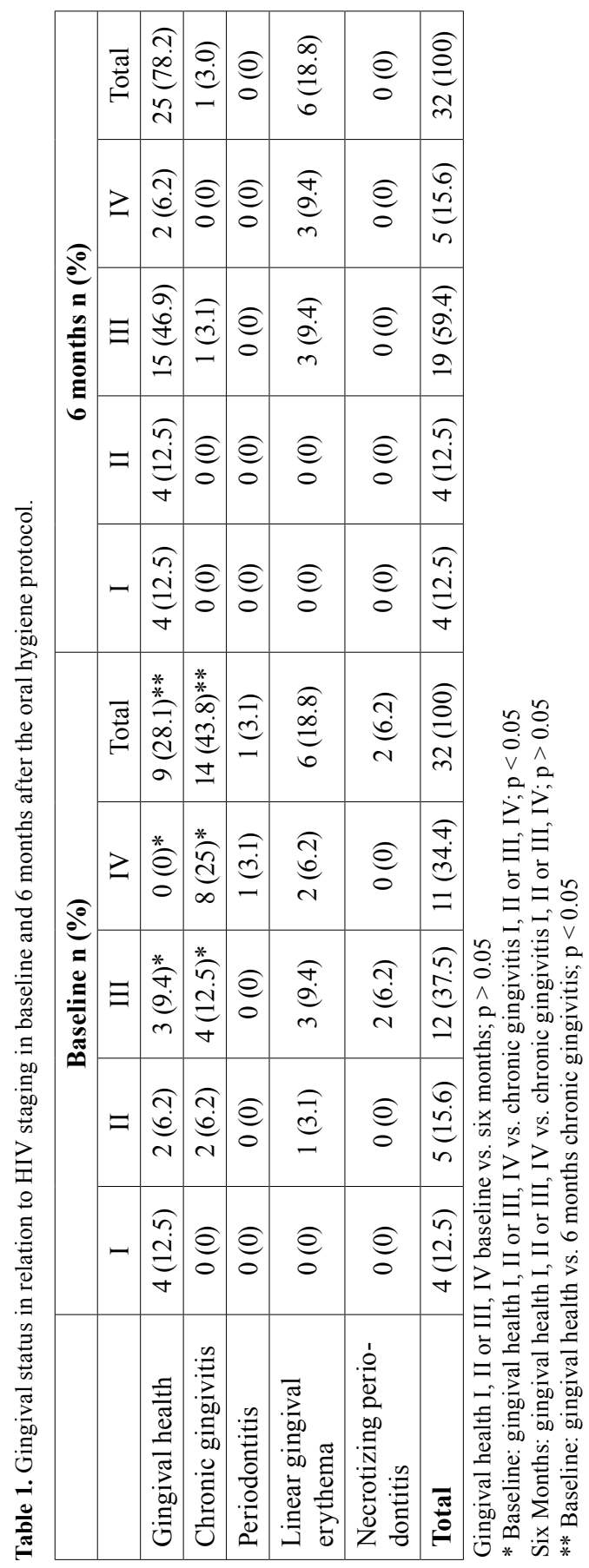


Table 2. Distribution of gingival status at baseline, and after 3 and 6 months of the oral hygiene protocol.

\begin{tabular}{|l|c|c|c|}
\hline Gingival status & $\begin{array}{c}\text { Baseline } \\
\mathrm{n}(\%)\end{array}$ & $\begin{array}{c}3 \text { months } \\
\mathrm{n}(\%)\end{array}$ & $\begin{array}{c}6 \text { months } \\
\mathrm{n}(\%)\end{array}$ \\
\hline Gingival health & $9(28.1)$ & $22(68.7)^{*}$ & $25(78.1)^{*}$ \\
\hline Chronic gingivitis & $14(43.8)$ & $3(9.4)$ & $1(3.1)$ \\
\hline Periodontitis & $1(3.1)$ & $0(0)$ & $0(0)$ \\
\hline Linear gingival erythema & $6(18.8)$ & $6(18.8)$ & $6(18.8)$ \\
\hline Necrotizing periodontitis & $2(6.2)$ & $1(3.1)$ & $0(0)$ \\
\hline Total & $32(100)$ & $32(100)$ & $32(100)$ \\
\hline Gingival index & & & \\
\hline $0.0-0.1$ & $9(28.1)$ & $22(68.7)^{* *}$ & $25(78.1)^{* *}$ \\
\hline $0.1-1.0$ & $7(21.9)$ & $6(18.8)$ & $5(15.7)$ \\
\hline $1.0-2.0$ & $13(40.6)$ & $3(9.4)$ & $1(3.1)$ \\
\hline $2.0-3.0$ & $3(9.4)$ & $1(3.1)$ & $1(3.1)$ \\
\hline Total & $32(100)$ & $32(100)$ & $32(100)$ \\
\hline Simplified Oral Hygiene Index (IHOS) & & & \\
\hline $0.0-1.2$ & $11(34.4)$ & $28(87.5)^{* * * *}$ & $28(87.6)^{* * *}$ \\
\hline $1.3-3.0$ & $9(28.1)$ & $1(3.1)$ & $2(6.2)$ \\
\hline $3.1-6.0$ & $12(37.5)$ & $3(9.4)$ & $2(6.2)$ \\
\hline Total & $32(100)$ & $32(100)$ & $32(100)$ \\
\hline$*$ Gingivar & & & \\
\hline
\end{tabular}

* Gingival health vs. Chronic Gingivitis $\mathrm{p} \leq 0.001$

Gingival health vs. Gingival Linear Erithema $p>0.05$

** 0.0-0.1 vs. $0.1-3.0 \mathrm{p} \leq 0.001$

*** $0.0-1.2$ vs. $1.3-6.0 \mathrm{p} \leq 0.001$

\section{Discussion}

In this study, mechanical therapy and oral hygiene maintenance improved the periodontal aspect in both HIV seropositive and AIDS patients. Chronic gingivitis was the most frequent periodontal disease in $43.8 \%$ of patients, contrary to other authors who have shown a frequency of $21.8 \%$ and $28 \%(2,19,20)$. Here, only one patient exhibited periodontitis, whereas other studies have shown up to $66 \%$ of attachment loss (6). HIV associated gingival disease represented $25 \%$. The frequency of aggressive periodontal disease in this study was low, despite the advanced stage of HIV infection. Other study has shown a range of 0 to $47 \%$ (5).

The systemic condition in HIV infection appears to be an important factor in the maintenance of periodontal status. The greatest frequency of periodontal disease was seen in patients with a more advanced stage of infection (Groups III and IV). Chronic gingivitis was the most prevalent in such patients, suggesting that the systemic condition in HIV infection does not necessarily predispose patients to pocketing or attachment loss.

No statistically significant association was registered between periodontal status and CD4 counts. This is in disagreement with another study (19). Gingival health was registered in subjects with low CD4 counts $(<500$ cells/mm3) after six months of mechanical therapy. No statistically significant association was found between bleeding on probing, pocket formation or attachment loss with CD4 counts among HIV infected individuals, suggesting that the improvement and maintenance of the gingival condition is not dependent on CD4 counts. In the present study, achieving gingival health after the oral hygiene protocol appeared to be an independent factor in relation to the CD4 count. These results suggest that immunodeficiency alone does not predispose patients to gingival and periodontal disease, since patients underwent an oral hygiene protocol. Other authors have also reported no consistent effect between periodontal status, HIV infection and CD4 count $(3,4,7,11,20)$.

A clear reduction in the gingival parameters and the improvement in the gingival condition in different HIV staging groups were evident after mechanical therapy. It is interesting to observe that improvement and maintenance of gingival status in AIDS patients was not dependent on HIV staging in the present study. It seems that oriented self care is the key point in the maintenance of oral hygiene in HIV infected subjects. These results are in agreement with those of other authors, who have achieved success after mechanical therapy in HIV infected patients $(2,7,11,12)$. 
In this study, the supportive periodontal therapy and the patient's cooperation in maintenance of oral hygiene improved the periodontal aspect in both HIV-seropositive and AIDS patients. There are some limitations which must be considered before the conclusions are drawn because this study was conducted in a closed population, represents a small sample and the non inclusion of a control group due to ethnical concepts by asking for HIV tests. However, study among HIV-infected and uninfected women showed no significant difference in the progression of periodontal disease (11).

In conclusion, chronic gingivitis was the most frequent disease in HIV infected and AIDS patients. Oral hygiene using mechanical therapy improves the gingival condition, suggesting that it is an important step in the maintenance of periodontal health. Achieving gingival health in the population studied does not seem to depend on immunodeficiency when an oral hygiene protocol is applied.

\section{References}

1. Holmstrup P, Glick M. Treatment of periodontal disease in the immunodeficient patient. Periodontol 2000. 2002;28:190-205.

2. Hofer D, Hämmerle CH, Grassi M, Lang NP. Long-term results of supportive periodontal therapy (SPT) in HIV-seropositive and HIVseronegative patients. J Clin Periodontol. 2002;29:630-7.

3. Scheutz F, Matee MI, Andsager L, Holm AM, Moshi J, Kagoma C, et al. Is there an association between periodontal condition and HIV infection? J Clin Periodontol. 1997;24:580-7.

4. Vastardis SA, Yukna RA, Fidel PL Jr, Leigh JE, Mercante DE. Periodontal disease in HIV-positive individuals: association of periodontal indices with stages of HIV disease. J Periodontol. 2003;74:1336-41.

5. Expósito-Delgado AJ, Vallejo-Bolaños E, Martos-Cobo EG. Oral manifestations of HIV infection in infants: a review article. Med Oral Patol Oral Cir Bucal. 2004;9:415-20, 410-5.

6. Aguirre-Urízar JM, Echebarría-Goicouría MA, Eguía-del-Valle A. Acquired immunodeficiency syndrome: manifestations in the oral cavity. Med Oral Patol Oral Cir Bucal. 2004;9 Suppl:153-7, 148-53.

7. Gonçalves Lde S, Ferreira SM, Silva A Jr, Villoria GE, Costinha
LH, Souto R, et al. Association of T CD4 lymphocyte levels and subgingival microbiota of chronic periodontitis in HIV-infected Brazilians under HAART. Oral Surg Oral Med Oral Pathol Oral Radiol Endod. 2004;97:196-203.

8. Heitz-Mayfield LJ. Disease progression: identification of high-risk groups and individuals for periodontitis. J Clin Periodontol. 2005;32 Suppl 6:196-209.

9. Westfelt E. Rationale of mechanical plaque control. J Clin Periodontol. 1996;23:263-7.

10. Robinson PG, Boulter A, Birnbaum W, Johnson NW. A controlled study of relative periodontal attachment loss in people with HIV infection. J Clin Periodontol. 2000;27:273-6.

11. Alves M, Mulligan R, Passaro D, Gawell S, Navazesh M, Phelan J, et al. Longitudinal evaluation of loss of attachment in HIVinfected women compared to HIV-uninfected women. J Periodontol. 2006;77:773-9.

12. Jordan RA, Gängler P, Jöhren HP. Clinical treatment outcomes of periodontal therapy in HIV-seropositive patients undergoing highly active antiretroviral therapy. Eur J Med Res. 2006;11:232-5.

13. Center of Disease Control and Prevention (CDC). Classification system for human T-lymphotropic virus type III/lymphadenopathyassociated virus infections. Morb Mortal Wkly Rep. 1986;35:334-9.

14. Grinsztejn B, Veloso VG, Pilotto JH, Campos DP, Keruly JC, Moore RD. Comparison of clinical response to initial highly active antiretroviral therapy in the patients in clinical care in the United States and Brazil. J Acquir Immune Defic Syndr. 2007;45:515-20.

15. No authors listed. Classification and diagnostic criteria for oral lesions in HIV infection. EC-Clearinghouse on Oral Problems Related to HIV Infection and WHO Collaborating Centre on Oral Manifestations of the Immunodeficiency Virus. J Oral Pathol Med. 1993;22:289-91.

16. Loe H, Silness J. Periodontal disease in pregnancy. I. Prevalence and severity. Acta Odontol Scand. 1963;21:533-51.

17. Greene JC, Vermillion JR. The simplified oral hygiene index: a method for classifying oral hygiene status. J Am Dental Assoc. 1960;61:172-9.

18. Ramfjord SP. The Periodontal Disease Index (PDI). J Periodontol. 1967;38:602-10.

19. Kroidl A, Schaeben A, Oette M, Wettstein M, Herfordt A, Häussinger D. Prevalence of oral lesions and periodontal diseases in HIV-infected patients on antiretroviral therapy. Eur J Med Res. 2005; 10:448-53.

20. Bravo IM, Correnti M, Escalona L, Perrone M, Brito A, Tovar V, et al. Prevalence of oral lesions in HIV patients related to CD4 cell count and viral load in a Venezuelan population. Med Oral Patol Oral Cir Bucal. 2006;11:E33-9. 